\title{
Entropy Associated with Local Stabilization of the Pulse Area in Multilevel Atomic Media
}

\author{
A.M. Alhasan* \\ Physics Department, Shaqra University, Shaqra 11961, Kingdom of Saudi Arabia
}

(Received June 13, 2017)

\begin{abstract}
This paper provides an entropy analysis to resonant and short pulses propagation in four-level atomic medium. As an example, we take $D_{1}$ transition in rubidium ${ }^{87} R b$ atoms including hyperfine structure. We show how to construct the time dependent Bloch-metric for each optical transition in the Liouville space. Furthermore, we attempt to relate local stabilization of the pulse area to the distribution of the space-entropy.
\end{abstract}

DOI: 10.12693/APhysPolA.133.1205

PACS/topics: 02.50.-r, 42.50.Gy, 42.50.Hz, 32.80.Qk, 32.10.Fn

\section{Introduction}

Propagation of ultra-short light pulses in two-level atomic media is often investigated via the McCall and Hahn area theorem $[1,2]$ and self-induced transparency (SIT) $[3,4]$. Alternatively, Lamb [5] showed that the equations of SIT can be considered within the standard equations of the inverse scattering transform (IST), the Zakharov-Shabat (ZS) eigenvalue problem [6]. The transient process of self-induced transparency soliton formation has been recently addressed [7]. In an attenuating media, the area theorem predicts the transparency for pulses with initial area of $2 \pi$. Using the Bäcklud transformations (BT) one can relate solution of the sine-Gordon (SG) equation to another. In addition, the breakup of pulses having initial area of multiple $2 \pi$ into separate $2 \pi$ pulses has been demonstrated $[8,5,9]$. The evidence of such breaking is studied through numerical integration of the coupled Maxwell-Bloch equations (MBE) [10, 11]. It has been shown that an oscillating tail will arise after the occurrence of breaking. This oscillating tail is often referred to as pulse radiation or ringing [12]. Recently, the propagation in atomic vapor has been reviewed [13]. It is worthwhile to state that the pulse breakup does not hold in general for propagation in multi-level atomic media [14]. Matusovsky et al. [15] attributed the absence and lack of sensitivity of the pulse break to the multiplicity of the levels and the unequal dipole moments of the allowed-dipole transition.

This paper presents an alternative view to the theoretical description of pulse propagation in atomic vapors. We are looking for entropy as an identifier for local stabilization of the pulse area, rather than its energy. The entropy has been recently addressed as a metric generator of dissipation in classical dissipative systems [16]. The relation between thermodynamic entropy and Shannon's measure of information are investigated in [17], where it has been

*e-mail: abuothman@su.edu.sa pointed out that entropy is defined on any probability distribution. Garbaczewski discussed entropy methods in connection with closed quantum systems [18]. In our paper, we are mainly concerned with space-entropy associated with the local stabilization of short pulses propagation.

Our treatment for the time evolution of the dressed atom is based upon the density matrix approach. We are working with the density matrix in the flatten form as $e_{\alpha}$, where $\alpha$ numerates the maximum accessible bases from 1 to 28 [19]. Such general formalism will enable us to reveal enhancements of all accessible multipoles of the density matrix.

It seems to us that it is difficult to define the pulse area for resonant interaction of strong ultra-short light pulses in four-level atom scheme, due to the finite duration of the pulses. Therefore, we are interested in short pulse propagation. Furthermore, we are motivated by the work on long pulse self-induced transparency [20]. Finally, we assume that the hyperfine structure is resolved and the multi-photon resonance approximation is satisfied, as the frequencies are tuned to their respective dipole allowed transitions. The initial phases of the fields are considered to be zero.

The paper is organized as follows. Section 2 introduces the relevant density matrix equations. The basic description of the Bloch-metric and the space-entropy are formulated, explained, and applied in Sect. 3. Sections 4 to 6 refer to results, discussions and conclusions.

\section{The theoretical description}

We analyze the coherent excitation of the $D_{1}$ line i.e. $5^{2} S_{1 / 2}-5^{2} P_{1 / 2}$ in ${ }^{87} \mathrm{Rb}$ atom where the hyperfine structure is taken into account as depicted in Fig. 1. The spontaneous decay rate of the excited $P_{1 / 2}$ state is given as $\gamma=5.746 \mathrm{MHz}$. The fields with the Rabi frequencies $\Omega_{p_{1}}$ and $\Omega_{p_{2}}$ couple the upper hyperfine levels $\left|3, F_{3}=1\right\rangle$ and $\left|4, F_{4}=2\right\rangle$ with the lower hyperfine level $\left|1, F_{1}=1\right\rangle$, respectively. In addition, the fields with the Rabi frequencies $\Omega_{c_{1}}$ and $\Omega_{c_{2}}$ couple the upper hyperfine levels $\left|3, F_{3}=1\right\rangle$ and $\left|4, F_{4}=2\right\rangle$ with the lower hyperfine level 
$\left|2, F_{2}=2\right\rangle$, respectively. The upper hyperfine splitting is $\Delta \omega_{4,3}=816.656 \mathrm{MHz}$ while the lower hyperfine splitting takes the value $\Delta \omega_{2,1}=6.834 \mathrm{GHz}$ [21]. These two frequency differences are assumed to be compensated by the two-photon resonance conditions as

$$
\Delta \omega_{4,3}=\omega_{p_{2}}-\omega_{p_{1}}, \quad \Delta \omega_{2,1}=\omega_{p_{1}}-\omega_{c_{1}} .
$$

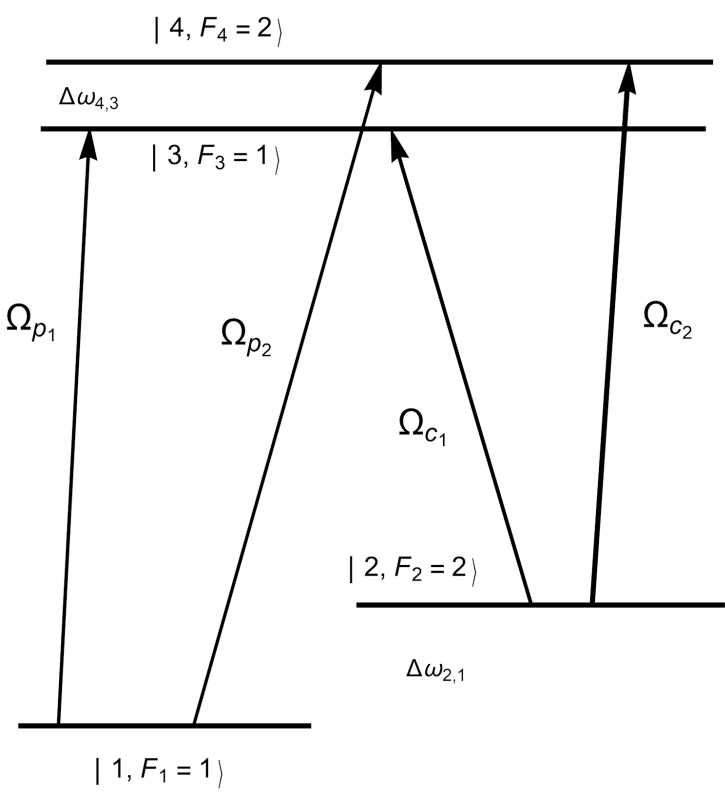

Fig. 1. The four-level scheme in a double $V$ or $\Lambda$ configuration. The pulses $\Omega_{p_{1}}$ and $\Omega_{p_{2}}$ drive the transitions $\left|3, F_{3}=1\right\rangle \leftrightarrow\left|1, F_{1}=1\right\rangle$ and $\left|4, F_{4}=2\right\rangle \leftrightarrow\left|1, F_{1}=1\right\rangle$, respectively. The pulses $\Omega_{c_{1}}$ and $\Omega_{c_{2}}$ couple the transitions $\left|3, F_{3}=1\right\rangle \leftrightarrow\left|2, F_{2}=2\right\rangle$ and $\left|4, F_{4}=2\right\rangle \leftrightarrow$ $\left|2, F_{2}=2\right\rangle$, respectively. The upper and lower hyperfine splitting are denoted by $\Delta \omega_{4,3}$ and $\Delta \omega_{2,1}$, respectively.

Our scheme presented in Fig. 1 shows a double electromagnetically induced transparency scheme. Windholz and his coworkers have investigated both theoretically and experimentally the coherent population trapping (CPT) [22-24].

In the present analysis for the double $\Lambda$ configuration, we shall assume zero value for the initial phases of the fields, in order to obtain a definition for real valued pulse area. Furthermore, we assume that the fields are linearly polarized and co-propagating where the sharp line limit (SLL) is to be considered. The spontaneous decay rates $\gamma_{u, l}$ with $u=\{3,4\}$ and $l=\{2,1\}$ are related to $\gamma$ by branching ratio. The state of the atom is described by Liouville-von Neumann equations for the density matrix of the dressed atom

$$
-\mathrm{i} \partial_{t} \rho=(\hat{H}+\mathrm{i} \hat{\Phi}) \rho, \hbar=1,
$$

where $\hat{H}$ and $\hat{\Phi}$ stand for the system Hamiltonian and the relaxation super-operators [25]. The relaxation superoperator contains both the radiation and collisional contributions [25]. For $D_{1}$ transition and linearly polarized fields, the density matrix is composed of 28 components [19]. These components are: four populations of the hyperfine levels, eight complex coherences, the align- ment components, upper- and lower-Raman coherences, components for the correlations between upper and lower hf-levels which are of third rank, and hexadecapole moment components. The number of equations is reduced to 19 for the case depicted in Fig. 1, as the on resonant condition is assumed. It is convenient to describe the density matrix components in the form $\rho_{i, j}^{(k)}$, where $k$ expresses the rank of the density matrix component and $i$ as well as $j$ numerate the hyperfine levels as shown in Fig. 1. However, we shall use the corresponding flattened form as $\rho_{\alpha}$, where $\alpha$ takes the values $1,2,3, \ldots 28$.

The correspondence between these two descriptions can be found with the aid of the collisional terms as listed in Appendix. In particular, we mention the population of the hyperfine levels which are proportional to the zero rank components: $\rho_{1} \leftrightarrow \rho_{1,1}^{(0)}, \rho_{2} \leftrightarrow \rho_{2,2}^{(0)}$, $\rho_{3} \leftrightarrow \rho_{3,3}^{(0)}$, and $\rho_{4} \leftrightarrow \rho_{4,4}^{(0)}$. The populations of the hf levels are denoted by

$$
n_{1}=\sqrt{3} \rho_{1}, n_{2}=\sqrt{5} \rho_{2}, n_{3}=\sqrt{3} \rho_{3}, n_{4}=\sqrt{5} \rho_{4} .
$$

The optical coherences for $\Omega_{p_{1}}$ and $\Omega_{p_{2}}$ transitions are denoted by $\rho_{5} \leftrightarrow \rho_{1,3}^{(1)}$ and $\rho_{7} \leftrightarrow \rho_{1,4}^{(1)}$, respectively, while the optical coherences corresponding to $\Omega_{c_{1}}$ and $\Omega_{c_{2}}$ transitions are denoted by $\rho_{9} \leftrightarrow \rho_{2,3}^{(1)}$ and $\rho_{11} \leftrightarrow \rho_{2,4}^{(1)}$, respectively. In addition, we have the second rank components such as: $\rho_{17} \leftrightarrow \rho_{1,2}^{(2)}, \rho_{19} \leftrightarrow \rho_{3,4}^{(2)}, \rho_{13} \leftrightarrow \rho_{1,1}^{(2)}$, $\rho_{14} \leftrightarrow \rho_{2,2}^{(2)}, \rho_{15} \leftrightarrow \rho_{3,3}^{(2)}$, and $\rho_{16} \leftrightarrow \rho_{4,4}^{(2)}$. The third rank components are described by $\rho_{21} \leftrightarrow \rho_{2,4}^{(3)}, \rho_{23} \leftrightarrow \rho_{2,3}^{(3)}$, and $\rho_{25} \leftrightarrow \rho_{1,4}^{(3)}$. Finally, we have the components with rank four as $\rho_{27} \leftrightarrow \rho_{2,2}^{(4)}$ and $\rho_{28} \leftrightarrow \rho_{4,4}^{(4)}$.

The density matrix equations are coupled to the reduced-Maxwell field equations in the rotating wave approximation. We shall follow our procedure with using relative units [19]. In order to write the reduced-Maxwell field equations in a frame moving with the pulse, we introduce the variables for space $\eta=z+c t$ and the retarded time $t^{\prime}=(t-z / c)$. So, we have $\partial_{\eta}=\partial_{z}+1 / c \partial_{t}$, and $c$ is the light speed here. Let us introduce the dimensionless variables for space and time as $\zeta=\alpha^{\prime} \eta$ and $\tau=\gamma t^{\prime}$, where $\alpha^{\prime}$ denotes the absorption coefficient of one of the pulses at $z=0$. The differences in absorption coefficients for the four transitions are ignored. The time duration of the pulse is described by $T_{p}$ and its dimensionless form as $T=\gamma T_{p}$. Finally, the atom-field coupling is denoted by $v=\frac{d_{r} E}{2 \sqrt{3}}$, where $d_{r}$ is the reduced dipole moment and $E$ is the electric field envelope. We have used $\hbar=1$. The atom-field coupling is denoted by $v_{\beta}$ and $\beta=\left\{p_{1}, p_{2}, c_{1}, c_{2}\right\}$. The Rabi frequency associated with the four transitions are given by $\Omega_{\beta}=\kappa v_{\beta}$, where $\kappa$ is the proportional constant, which is to be determined later. The dimensionless atom-field coupling is denoted by $\mathrm{v}_{\beta}=v_{\beta} / \gamma$.

The reduced-Maxwell field equations in a frame moving with the pulse can be written as

$$
\partial_{\zeta} \mathrm{v}_{p_{1}}(\zeta, \tau)=-\rho_{5}(\zeta, \tau),
$$




$$
\begin{gathered}
\partial_{\zeta} \mathrm{v}_{p_{2}}(\zeta, \tau)=\rho_{7}(\zeta, \tau), \\
\partial_{\zeta} \mathrm{v}_{c_{1}}(\zeta, \tau)=-\rho_{9}(\zeta, \tau), \\
\partial_{\zeta} \mathrm{v}_{c_{2}}(\zeta, \tau)=\rho_{11}(\zeta, \tau) .
\end{gathered}
$$

At the entrance boundary $z=0$ we assume that the temporal pulse profile takes the form of $t^{2}$-Gaussian shape as

$$
v(t)=\frac{64 \sqrt{2} \pi}{27} v_{0}\left(t / T_{p}\right)^{2} e^{-8 / 9 \pi\left(t / T_{p}\right)^{2}} .
$$

The pulse is characterized by its mean amplitude $v_{0}$ and mean duration $T_{p}$ as

$$
v_{0}=\int_{0}^{\infty} v^{2}(t) \mathrm{d} t / \int_{0}^{\infty} v(t) \mathrm{d} t
$$

and

$$
T_{p}=\left(\int_{0}^{\infty} v(t) \mathrm{d} t\right)^{2} / \int_{0}^{\infty} v^{2}(t) \mathrm{d} t .
$$

The pulse area is defined as the integral of the field envelope over time:

$$
\theta(z)=\kappa \int_{0}^{\infty} v(t) \mathrm{d} t
$$

We may anticipate our results and take $\kappa$ as

$$
\kappa=\sqrt{8}
$$

In our analysis we have restricted ourselves to rapidly increasing pulse at its front, and a decaying Gaussian shape for its tail.

\section{Rate of change of metric tensor and Bloch-metric}

In this section we will give a brief account of entropy that is seemed to be useful in the analysis of resonant pulses propagation. Furthermore, it gives a signature for local stabilization of pulses as well as its breaking. We attempt to describe these effects in terms of the Shannon entropy and analogously to the Rènyi entropy. Entropy has been used for detecting dynamical changes in time series for complex systems [26]. Vardi and Shapiro showed entropy exchange between matter and radiation using time dependence of the linear Rènyi entropy [27]. The rate of entropy production due to absorption and emission of photons has been discussed in [28]. The entropy production associated with quantum jumps has been studied in [29].

The Bloch space structure with eight-dimensional Bloch sphere for three-level systems has been introduced using $S U(3)$ basis [30]. The Bloch sphere is shown to be divided into two sectors. The geometry of the generalized Bloch sphere for qutrit has been reported in [31]. For the present analysis we have four-level atom structure.

The Bloch vector $\operatorname{Tr} \rho^{2}$ is to be found subject to $\operatorname{Tr} \rho=1$ constraint on the density matrix components. The geometry of the Bloch sphere is complicated, since it is composed of 28 components, in general. In the present analysis we will decompose the Bloch geometry into four two-level atoms. Each one of them corresponds to the relevant optical transition with restricted components such as the population inversion among the hf-levels and the optical coherences. Then, the goal is to find the Liovillian which generate the space of the associated optical transition. Furthermore, the Bloch vector $B^{2}(t)$ is obtained in an algebraic technique through the subspace components generated by the Liouvillian. We simply avoid $\operatorname{Tr} \rho=1$ for each subspace by establishing the escort probability $[32,33]$ density as

$$
p(t)=\frac{B^{2}(t)}{\sum_{t^{\prime}}^{t} B^{2}\left(t^{\prime}\right)} .
$$

The procedure is outlined in the following section. It is to be emphasized that our definition is different from that used by Bercher [33], where $B(t)$ is not the original distribution with the condition $\sum_{t} B(t)=1$.

\subsection{Construction of space-entropy for each optical transition}

Let us consider the first subsystem characterized by the Liouvillian $\mathcal{L}_{1}$ as

$$
\mathcal{L}_{1}=\left(\begin{array}{cccc}
0 & \frac{\gamma}{6} & -\frac{v_{p_{1}}}{\sqrt{3}} & -\frac{v_{p_{1}}}{\sqrt{3}} \\
0 & -\gamma & \frac{v_{p_{1}}}{\sqrt{3}} & \frac{v_{p_{1}}}{\sqrt{3}} \\
\frac{v_{p_{1}}}{\sqrt{3}} & -\frac{v_{p_{1}}}{\sqrt{3}} & -\Gamma & 0 \\
\frac{v_{p_{1}}}{\sqrt{3}} & -\frac{v_{p_{1}}}{\sqrt{3}} & 0 & -\Gamma
\end{array}\right)
$$

connecting the ground hyperfine state $|1\rangle=\left|F_{1}=1\right\rangle$ with the upper hf state $|3\rangle=\left|F_{3}=1\right\rangle$. The Liouvillian is composed from the coupling field $v_{p_{1}}$ as the first generator of $\mathcal{L}_{1}$ subspace, and the relaxation $\Phi_{1}$ operator as the second generator of $\mathcal{L}_{1}$ subspace. One of these generators is sufficient to generate the bases. The relaxation $\Phi_{1}$ super-operator can be written as

$$
\Phi_{1}=\left(\begin{array}{cccc}
0 & \frac{\gamma}{6} & 0 & 0 \\
0 & -\gamma & 0 & 0 \\
0 & 0 & -\Gamma & 0 \\
0 & 0 & 0 & -\Gamma
\end{array}\right) .
$$

The space for the first subsystem is spanned by four density matrix components with a tensor

$$
\rho_{p_{1}}=\left\{\rho_{1}, \rho_{3}, \rho_{5}, \rho_{6}\right\},
$$

and time evolution

$$
\partial_{t} \rho_{p_{1}}=\mathcal{L}_{1} \rho_{p_{1}} .
$$

The metric tensor of this subspace is given as

$$
\rho_{p_{1}}^{2}=\rho_{p_{1}}^{\mathrm{T}} \cdot \rho_{p_{1}}=\rho_{1}^{2}+\rho_{3}^{2}+\rho_{5}^{2}+\rho_{6}^{2} .
$$

The rate of change of the metric tensor for $\mathcal{L}_{1}$ subspace is given by

$$
\begin{gathered}
\frac{1}{2} \frac{\partial}{\partial t} \operatorname{Tr} \rho_{p_{1}}^{2}=\operatorname{Tr}\left\{\rho_{p_{1}}^{T} \cdot\left(\Phi_{1} \rho_{p_{1}}\right)\right\}= \\
-\gamma \rho_{3}^{2}+\frac{1}{6} \gamma \rho_{1} \rho_{3}-\Gamma\left(\rho_{5}^{2}+\rho_{6}^{2}\right)= \\
\gamma \rho_{3}\left(\frac{\rho_{1}}{6}-\rho_{3}\right)-\Gamma\left(\rho_{5}^{2}+\rho_{6}^{2}\right) .
\end{gathered}
$$


From Eq. (19), the weighted population inversion within the first subsystem may be written as $w=\left(\frac{\rho_{1}}{6}-\rho_{3}\right)$. This is not true, since the terms $\partial_{t} w^{2}$ and $\partial_{t}\left(\rho_{5}^{2}+\rho_{6}^{2}\right)$ are not compensated to produce traceless time variation of the Bloch vector over the field. We mean by weighted population inversion that one which is explicitly dependent on the branching ratio of the excited hf levels. Let us construct the weighted population inversion and the Bloch squared length for the Bloch vector of the first subsystem as

$W_{1}=\left(\rho_{3}-\rho_{1}\right), \quad B_{1}^{2}=\frac{1}{2}\left(\rho_{3}-\rho_{1}\right)^{2}+\left(\rho_{5}^{2}+\rho_{6}^{2}\right)$.

The squared length of the Bloch vector is composed from the population inversion and coherence terms

$$
\frac{1}{2} \partial_{t} B_{1}^{2}=\frac{7}{12} \gamma \rho_{3}\left(\rho_{1}-\rho_{3}\right)-\Gamma\left(\rho_{5}^{2}+\rho_{6}^{2}\right)
$$

We shall denote the constructed squared length of the Bloch vector as the Bloch-metric. So, rate of change of the Bloch-metric is traceless over the field $v_{p_{1}}$ which imposes the correctness of our choice for the population inversion. This choice is identical with the instantaneous population inversion between the first and third hf level. Since, these states have the same statistical weight.

The states $|1\rangle=\left|F_{1}=1\right\rangle$ and $|4\rangle=\left|F_{4}=2\right\rangle$ form the optical transition for the second subsystem with coupling field $v_{p_{2}}$. The hf states $|1\rangle$ and $|4\rangle$ have different statistical weights. For the second subsystem the Liouvillian is written as

$$
\mathcal{L}_{2}=\left(\begin{array}{cccc}
0 & \frac{1}{2} \sqrt{\frac{5}{3}} \gamma & \sqrt{\frac{5}{3}} v_{p_{2}} & \sqrt{\frac{5}{3}} v_{p_{2}} \\
0 & -\gamma & -v_{p_{2}} & -v_{p_{2}} \\
-\sqrt{\frac{5}{3}} v_{p_{2}} & v_{p_{2}} & -\Gamma & 0 \\
-\sqrt{\frac{5}{3}} v_{p_{2}} & v_{p_{2}} & 0 & -\Gamma \\
, & & &
\end{array}\right)
$$

where the density matrix

$$
\rho_{p_{2}}=\left(\rho_{1}, \rho_{4}, \rho_{7}, \rho_{8}\right)
$$

spans the second subspace from the total Liouville space with time evolution

$$
\partial_{t} \rho_{p_{2}}=\mathcal{L}_{2} \rho_{p_{2}} .
$$

Let us assume that the Bloch-metric for the second subsystem can be factorized as

$$
B_{2}^{2}=\frac{1}{2} a\left(c \rho_{4}-\rho_{1}\right)^{2}+\rho_{7}^{2}+\rho_{8}^{2},
$$

where $a$ and $c$ are constants, yet to be determined. The constants $a$ and $c$ which insure the traceless of the dependence in $B_{2}^{2}$ on $v_{p_{2}}$ are: $a=5 / 4$ and $c=3 / 5$. Therefore

$$
W_{2}=\sqrt{\frac{3}{5}} \rho_{4}-\rho_{1}, \quad B_{2}^{2}=\frac{5 W_{2}^{2}}{8}+\rho_{7}^{2}+\rho_{8}^{2} .
$$

The first two subsystems form the $v_{p}$ branch connecting the first ground hf level to the excited and upper hyperfine levels. The other $v_{c}$ branch is connecting the second and ground hf level with the excited and upper hf levels. The third subsystem optical transition is the transition $|2\rangle=\left|F_{2}=2\right\rangle$ with the upper hf state $|3\rangle=\left|F_{3}=1\right\rangle$ while the driving field is $v_{c_{1}}$. Let $\rho_{c_{1}}$ with the components $\rho_{c_{1}}=\left(\rho_{2}, \rho_{3}, \rho_{9}, \rho_{10}\right)$ represent the generators for the third $v_{c_{1}}$ subspace with

$$
\mathcal{L}_{3}=\left(\begin{array}{cccc}
0 & \frac{1}{2} \sqrt{\frac{5}{3}} \gamma & -v_{c_{1}} & -v_{c_{1}} \\
0 & -\gamma & \sqrt{\frac{5}{3}} v_{c_{1}} & \sqrt{\frac{5}{3}} v_{c_{1}} \\
v_{c_{1}} & -\sqrt{\frac{5}{3}} v_{c_{1}} & -\Gamma & 0 \\
v_{c_{1}} & -\sqrt{\frac{5}{3}} v_{c_{1}} & 0 & -\Gamma \\
\cdot & & &
\end{array}\right)
$$

With the same procedure outlined previously for the case of different statistical weight for levels, we can write for the population inversion and the squared length for the Bloch vector as

$$
\begin{aligned}
& W_{3}=\left(\sqrt{\frac{5}{3}} \rho_{3}-\rho_{2}\right) \\
& B_{3}^{2}=\frac{3}{8}\left(\sqrt{\frac{5}{3}} \rho_{3}-\rho_{2}\right)^{2}+\rho_{9}^{2}+\rho_{10}^{2} .
\end{aligned}
$$

Let $\rho_{c_{2}}$ with the components $\rho_{c_{2}}=\left(\rho_{2}, \rho_{4}, \rho_{11}, \rho_{12}\right)$ represent the generators for the fourth $v_{c_{2}}$ subspace with

$$
\mathcal{L}_{4}=\left(\begin{array}{cccc}
0 & \frac{\gamma}{2} & v_{c_{2}} & v_{c_{2}} \\
0 & -\gamma & -v_{c_{2}} & -v_{c_{2}} \\
-v_{c_{2}} & 0 & -\Gamma & 0 \\
-v_{c_{2}} & 0 & 0 & -\Gamma \\
\cdot & & &
\end{array}\right)
$$

and the population inversion as well as the squared length of the Bloch vector can be written as

$W_{4}=\left(\rho_{4}-\rho_{2}\right), \quad B_{4}^{2}=\frac{1}{2}\left(\rho_{4}-\rho_{2}\right)^{2}+\rho_{11}^{2}+\rho_{12}^{2}$

Finally, let us construct the probability density functions with respect to time and at fixed space point $z$ as

$$
p_{\alpha}(z, t)=\frac{B_{\alpha}^{2}(z, t)}{\sum_{t^{\prime}=t_{0}}^{t^{\prime}=t_{f}} B_{\alpha}^{2}\left(z, t^{\prime}\right)},
$$

where $\alpha$ takes the values 1 up to 4 , representing the first, second, third, and fourth subsystems, respectively. The initial and finial times are denoted by $t_{0}$ and $t_{f}$, respectively. We termed

$$
S_{\alpha}(z)=-\sum_{t=t_{0}}^{t=t_{f}} p_{\alpha}(z, t) \log _{2}\left(p_{\alpha}(z, t)\right)
$$

as the space-entropy which represents the Shannon entropy associated with the normalized Bloch squared length probability density function. The Bloch entropy is considered as a generator for population inversion and dissipation out of the atomic system under consideration. The quantum interference between these two terms leads to area construction (build-up of area) or destruction. 


\section{Results}

This section provides an entropy analysis for short pulses propagation in a four-level atomic medium. The optical pulse is resonant to one of the four atomic transitions as indicated in Fig. 1. The propagation dynamics is given by the numerical solution of the coupled density matrix components, listed in Appendix, and the reduced Maxwell field equations: Eq. (4) to Eq. (7). The configuration in Fig. 1 is discussed in terms of double $V$ subsystems. The first $V_{1}$ subsystem connects excited hf states $|3\rangle$ and $|4\rangle$ to the first ground hf state $|1\rangle$, while the second $V_{2}$ subsystem connects excited hf states $|3\rangle$ and $|4\rangle$ to the second ground hf state $|2\rangle$.

\subsection{Space-entropy resonance lines for $V_{1}$ subsystem}

4.1.1. Propagation dynamics for space-entropy $S_{2}(\zeta)$ associated with the field $\Omega_{p_{2}}(\tau)$ and the optical transition $|1\rangle \leftrightarrow|4\rangle$

Space-entropy (Eq. (32)) is illustrated in Fig. 2 for $\Omega_{p_{2}}$ transition and for different initial pulse area $\theta_{0}=\theta(\zeta=$ $0)$ as $\theta_{0}=\{3,9,2,8\}$ in $\pi$ units. We have restricted our discussions to the case when the four pulses have the same initial area $\theta_{0}$. We take the notations

$$
\begin{aligned}
& \theta_{\mathrm{v}_{p_{1}}}(\zeta)=\int_{0}^{\tau_{\max }} \sqrt{8} \mathrm{v}_{p_{1}}(\zeta, \tau) \mathrm{d} \tau, \\
& \theta_{\mathrm{v}_{p_{2}}}(\zeta)=\int_{0}^{\tau_{\max }} \sqrt{8} \mathrm{v}_{p_{2}}(\zeta, \tau) \mathrm{d} \tau, \\
& \theta_{\mathrm{v}_{c_{1}}}(\zeta)=\int_{0}^{\tau_{\max }} \sqrt{8} \mathrm{v}_{c_{1}}(\zeta, \tau) \mathrm{d} \tau, \\
& \theta_{\mathrm{v}_{c_{2}}}(\zeta)=\int_{0}^{\tau_{\max }} \sqrt{8} \mathrm{v}_{c_{2}}(\zeta, \tau) \mathrm{d} \tau,
\end{aligned}
$$

and $\tau_{\max }=10$, which is the maximum time for the time variable. In Fig. 2 the space-entropy $S_{2}(\zeta)$ resonance lines are exposed, where the horizontal base line is the maximum entropy. The maximum entropy is independent of the initial pulse area $\theta_{0}$ for big distances.

4.1.2. Propagation dynamics for the area $\theta_{2}(\zeta)$ associated with the field $\Omega_{p_{2}}(\tau)$ and the optical transition $|1\rangle \leftrightarrow|4\rangle$

The space dependence of pulse area $\theta_{2}(\zeta)$ is depicted in Fig. 3, where the local stabilization of $2 m \pi$ pulses is shown. The number $m$ is an integer number and $m \geq 2$. We mean by local stabilization of area that the area is merely constant over some prolonged distance. The value $2 m \pi$ is also the critical value for pulses breakdown to a value $2(m-1) \pi$. By inspection of Fig. 4 , we conclude that the drops of space-entropy are due to area-transitions by a $2 \pi$ value. $S_{2}(\zeta)$ shows these transitions at $2 m \pi$ values. These transitions are accomplished by area nutation nearby the critical $2 m \pi$ values showing direction towards the nearest local stabilization.

The space-width in the local stabilization for the area corresponds to maximum space-entropy in $S_{2}(\zeta)$. In addition, all transitions are bounded from above by the maximum-entropy. Figure 4 shows that the local stabilization width in space for the area can be estimated from
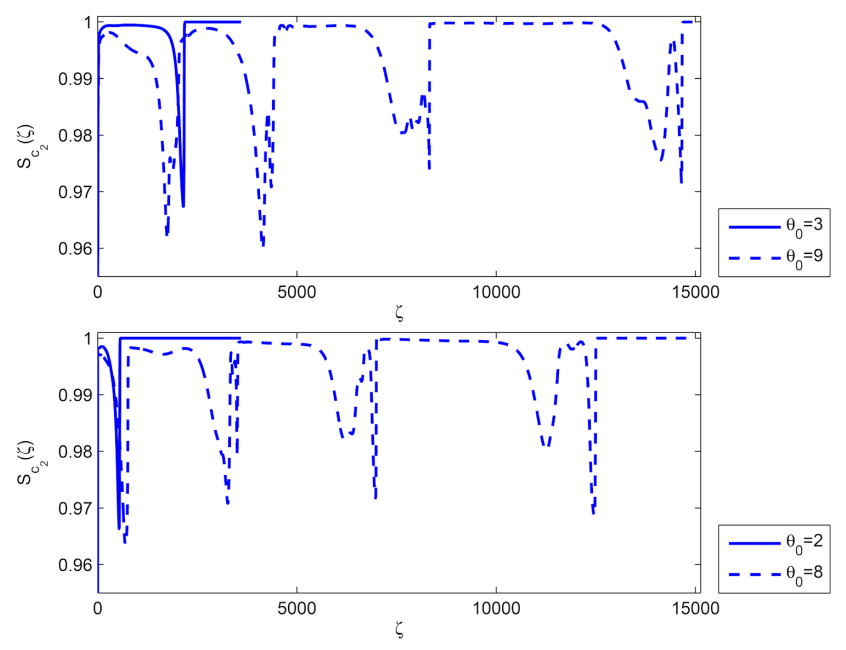

Fig. 2. Space-entropy resonance lines for $\Omega_{p_{2}}$ transition.

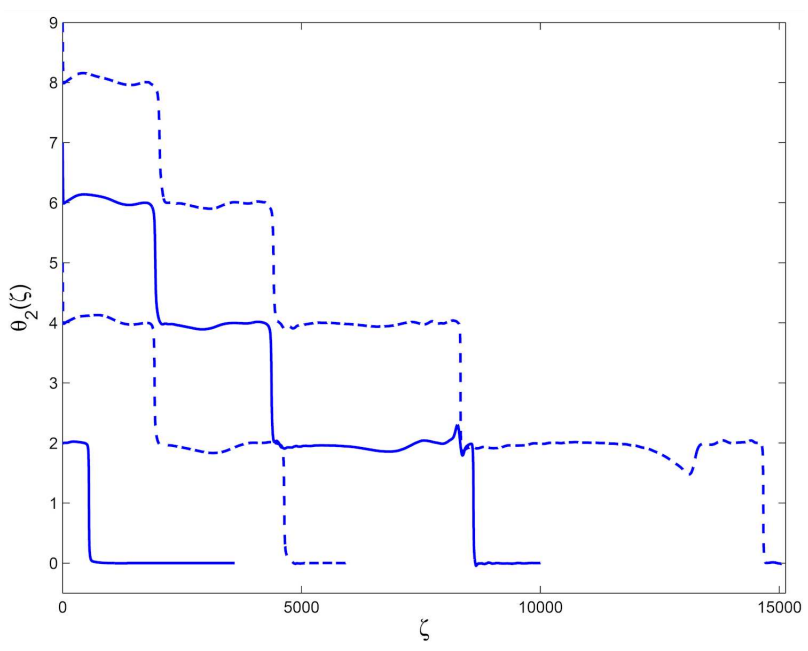

Fig. 3. Local stabilization of $2 m \pi$ pulses associated with $\Omega_{p_{2}}$ second transition. The number $m$ is an integer such that $m \geq 2$. The dependence of stabilization on the initial area are shown from above to below as $\theta_{0}=9,7,5$, and 2 in $\pi$ units.

the width in space for maximum-entropy. The stabilization width means the distance over which the area of the pulses in space $\theta(\zeta)$ becomes nearly constant. Figure 3 shows that the space width for the propagated $2 \pi$ pulse has the largest stabilization-width in space.

It is amazing to note that the structure in Fig. 3 showing the area transitions from $2 m \pi$ to $2(m-1) \pi$ occur not only regularly, but also at nearly the same position in space for different initial area. This indicates that the area parameter for the second transition could be used to predict the space dependence of the information in the experimental work. The slight nutation in area presented in Fig. 3 is transferred into variations toward the direction of increasing entropy to reach maximum-entropy and to obtain next nearby stabilization (Fig. 3). 


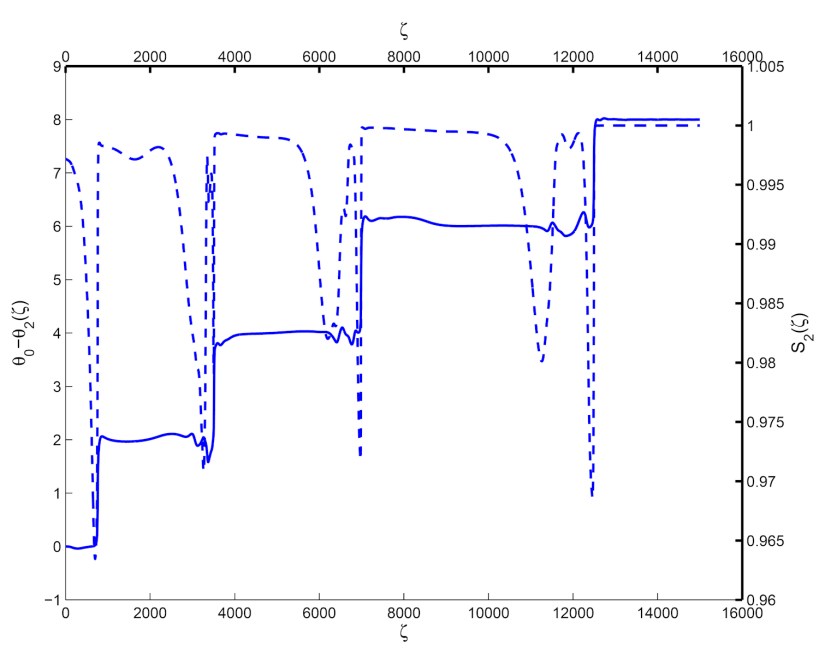

Fig. 4. Space-entropy transitions and pulse area for the second transition in the course of propagation. The initial area is taken as $\theta_{0}=8$ in $\pi$ units. The solid line represents $\theta_{0}-\theta_{2}(\zeta)$ and the dotted line represents $S_{2}(\zeta)$

These fluctuations in space-entropy around the critical values, and the area nutation make the area not a good dynamical variable for space-entropy considerations. Therefore, one cannot explicitly express $S_{2}=$ $S_{2}\left(\theta_{2}\right)$. Since, near critical values the space-entropy is no longer single valued function. This behavior is depicted in Fig. 5. Discussions concerning single and multivalued transformations have been pointed out by Robinson $[34,35]$.
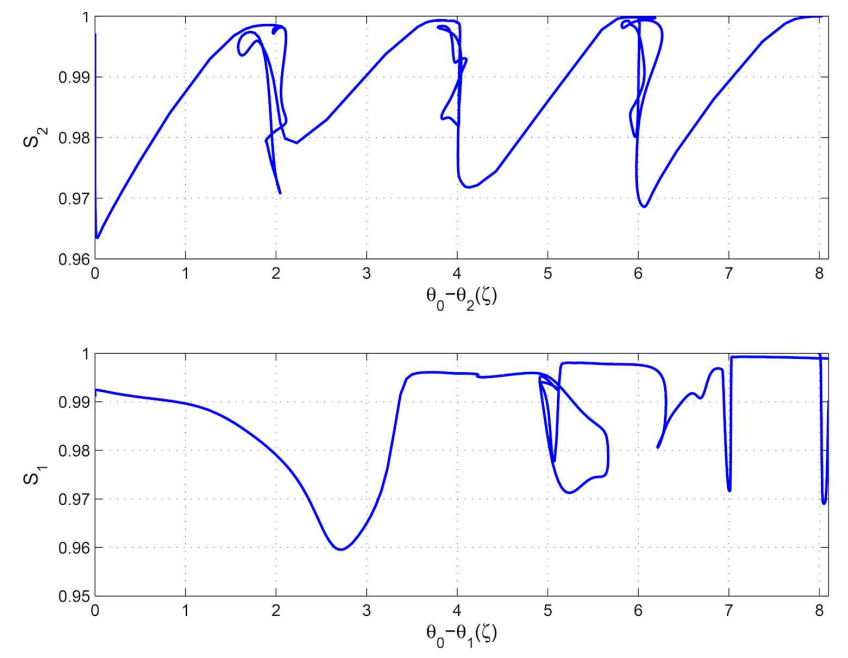

Fig. 5. Space-entropy transitions against pulse area for the first and second transition. The initial area is taken as $\theta_{0}=8$ in $\pi$ units.

4.1.3. Propagation dynamics for space-entropy $S_{1}(\zeta)$ associated with the field $\Omega_{p_{1}}(\tau)$ and the optical transition $|1\rangle \leftrightarrow|3\rangle$

The $\theta_{1}(\zeta)$ behavior looks like the weak field limit of the two-level atom. This is in the sense that the area more or less attenuates in the course of propagation. It is evident from Fig. 5 that $S_{1}(\zeta)$ does not show sharp transitions about the values $2 m \pi$ where $m$ is an integer. Some of these transitions are near $\pi$ and $3 \pi$ for relatively weak and moderate areas. For strong areas, $S_{1}\left(\theta_{1}\right)$ shows a smooth function of area with one minimum. These odd- $\pi$ transitions are smeared out in the space behavior of $\theta_{1}(\zeta)$. The area $\theta_{1}(\zeta)$ is a continuous decaying function in space without dips that is showing transition to lower area values. While space-entropy identifies these smeared-out resonances as these transitions are not stable.

\subsection{Space-entropy resonance lines for $V_{2}$ subsystem}

In this section we shall be concerned with the propagation dynamics and space entropy consideration for the second $V_{2}$ subsystem. This $V_{2}$ subsystem is connected to the first $V_{1}$ subsystem through the excited states $|3\rangle$ and $|4\rangle$. Furthermore, both of them share the common upper-Raman coherence. Additionally, the second subsystem has its own third rank components connecting the excited states $|3\rangle$ and $|4\rangle$ to second ground hf state $|2\rangle$. Figure 6 presents the space-entropy $S_{3}(\zeta)$ and $S_{4}(\zeta)$ for the third and fourth transitions, respectively, where the initial area is taken as $\theta_{0}=8$.

The third transition connecting the hf states $|2\rangle$ and $|3\rangle$ join states with different statistical weights. We have deduced its squared length for the Bloch vector as $B_{3}^{2}$ in Eq. (28). The fourth transition connects states $|4\rangle$ and $|2\rangle$ and these states have the same statistical weight. For the fourth transition the squared length for its Bloch vector was given as $B_{4}^{2}$ in Eq. (30). To our understanding, concerning propagation dynamics for connected levels with the same statistical weight, we may expect that the fourth transition is less stabilized than the third transition. This feature is revealed by inspection of the behavior of $S_{3}(\zeta)$ and $S_{4}(\zeta)$ in Fig. 6 . The space-entropy reduction in the fourth transition is exposed. The distinct critical area as $2 m \pi$ are no longer valid for spacetransitions in Fig. 6. The space-transitions appear with fractional values in area. The behavior of $\theta_{3}(z)$ shows that the field $\Omega_{c_{1}}$ is amplified in the course of propagation, while $\Omega_{c_{2}}$ is attenuated and its area becomes negative for big distance.

\subsection{Space-entropy associated with negative area propagation}

In this section we pay attention to propagation with negative area. As example, let $\theta_{0}=4$ in units of $\pi$. Figure 7 shows the envelope functions of $v_{c_{1}}(\tau)$ and $v_{c_{2}}(\tau)$ at $z \approx 6000$. These shape functions for the second $V_{2}$ subsystem. The fields on the $V_{1}$ subsystem are relatively small. Thus, the propagation dynamics collapses to propagation into $V_{2}$ subsystem with upper-Raman coherence. It is worth mentioning that negative area does not conclusively mean negative field envelope. But some negative area propagation is produced by a long tail contribution with negative area which dominates over the front of the pulse. 

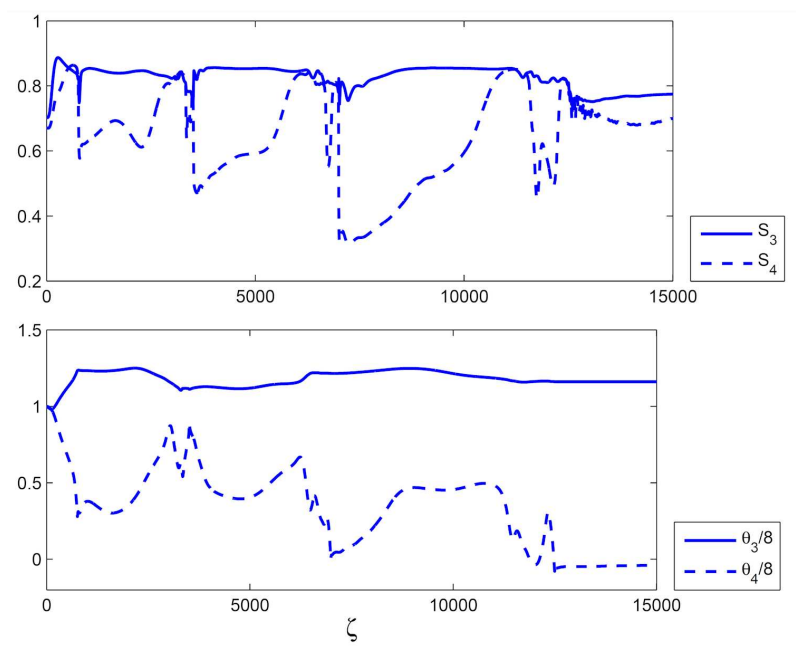

Fig. 6. Space-entropy of $S_{3}(\zeta)$ and $S_{4}(\zeta)$ for the third and fourth transitions, respectively.
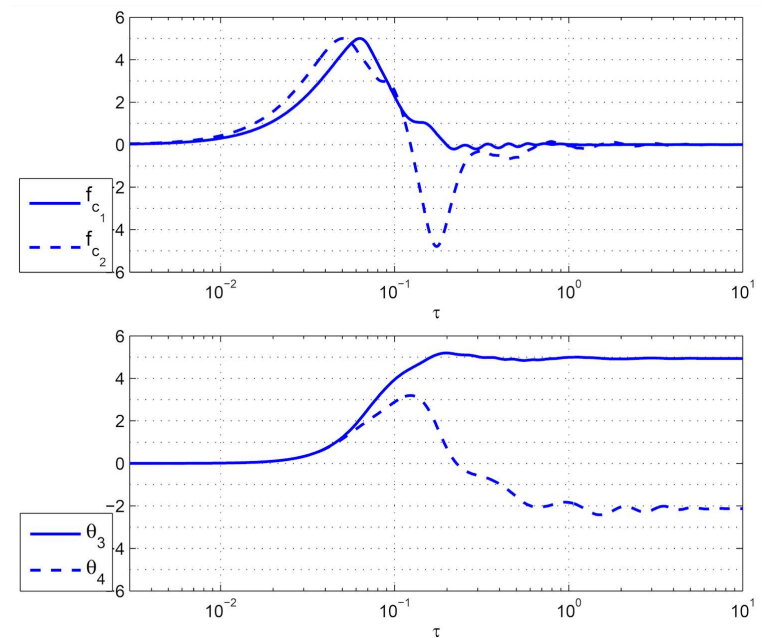

Fig. 7. Time dependence of envelope functions and the area $\theta_{3}$ as well as $\theta_{4}$ for the third and fourth transitions, respectively. The area represented in $\pi$ units. The envelope functions $f_{c_{1}}=v_{c_{1}}(t) / \max \left(v_{c_{1}}(t)\right)$ and $f_{c_{2}}=v_{c_{2}}(t) / \max \left(v_{c_{2}}(t)\right)$ show the relative temporal behavior of the pulses: $v_{c_{1}}(t)$ and $v_{c_{2}}(t)$. They are guideline eye to show the front and tail of the pulse. $z \approx 6000$ and $\theta_{0}=4$ in $\pi$ units..

The stabilized area is composed of three soliton-like envelope shapes with kink and anti-kink, which produce zero area as in Fig. 7. The third soliton-like shape is a generated tail with mainly negative envelope and area. Figure 6 presents the space-entropy for the second $V$ subsystems with space-entropy components $\left\{S_{3}(\zeta), S_{4}(\zeta)\right\}$. The space entropy $S_{4}$ has the largest dip or equivalently $\Delta S_{4}$ is maximum, characterizing a propagation with negative area.

Figure 8 shows the space-entropy $S_{3}$ as well as the area $\theta_{3}(\zeta)$ in the course of propagation. In the period when the area approaching the zero value from left and from right, the space-entropy approaches the minimum entropy. Finally, the area stabilizes at the negative level of $\approx 2 \pi$.

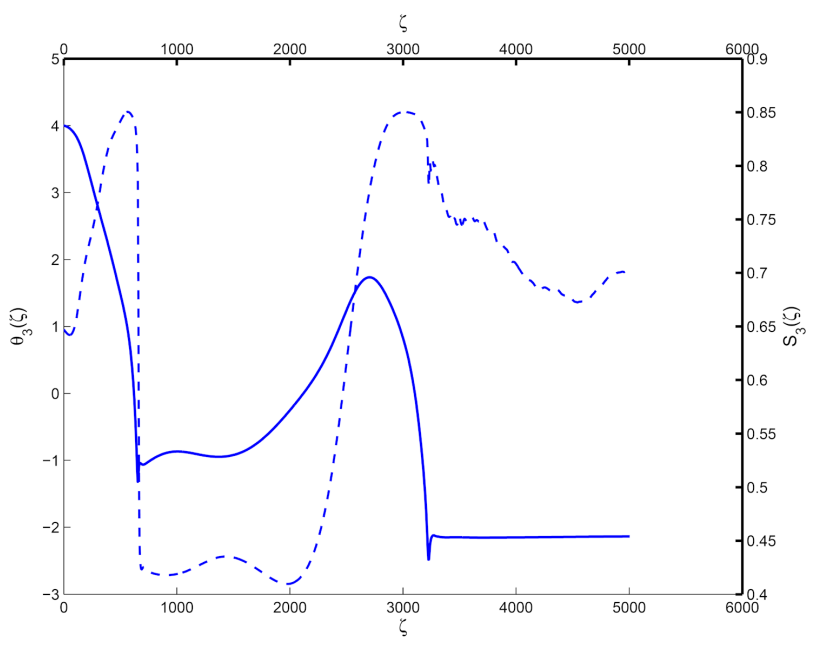

Fig. 8. Space-entropy for $S_{3}(\zeta)$ (dashed line) as well as the area $\theta_{3}(\zeta)$ (solid line) in the course of propagation..

\section{Discussions}

Our treatment for the propagation dynamics of short pulses in multilevel atomic media is connected to the entropy concept. This approach is suitable for the area local stabilization irrespective of energy stabilization. In the present study, the local energy fails to justify the local stabilization in the pulses area. The simple case where the energy stabilization leads to area stabilization and soliton propagation is no longer adequate in our present study. Strictly speaking the condition on energy stabilization in terms of area does not exist. The multiple light storage effects are significant in our present study. The multiple light storage effects manifest itself in area production and destruction in the course of propagation. In the present study, the area of the waveform is a result of interference between kinks and anti-kinks waveforms. Therefore the simple mapping of coherence in terms of area does not exist. We have appealed to the entropy concept through we have termed as the space-entropy in order to find something else other than energy which justifies and identifies the local stabilization.

In fact, if we pay attention to entropy or not, the basic constraints on the solution of the Maxwell-Bloch equations (MBE's) are not avoidable. These constraints are on $\operatorname{Tr} \rho$ and $\operatorname{Tr} \rho^{2}$. The Bloch entropy introduced is some function on $\operatorname{Tr} \rho^{2}$ in general. In any case without the condition on $\frac{1}{2} \partial_{t} \operatorname{Tr} \rho^{2}$ one cannot find analytical solution to the reduced Maxwell-Bloch equations (RMBE's) for a simple two level atom case and ignoring the atomic relaxation. Therefore, the treatment of entropy in our paper is natural. 


\section{Conclusions}

In this study, we have restricted ourselves to the case where the pulses have the same initial area and time duration. The pulses have initially the same shape as $t^{2}$ Gaussian. We have facilitated a dynamical entropy approach to describe the space-entropy. It is generated by the irreducible tensorial components of the statistical operator for $D_{1}$ transition in ${ }^{87} R b$. The statistical weight for the locally stabilized area is found to be $\kappa=\sqrt{8}$, which is independent of the transition.

In this study, we have shown the dependence of area and space-entropy upon the initial the area $\theta_{0}$ of the propagating pulses. It is assumed that the initial area takes the values $\theta_{0}=2 m \pi$ where $m$ is an integer.

1. We have reported propagation in one channel among the four two-level atomic channels, which is shown to be in a good resemblance to the area theorem.

2. The local stabilization of $2 m \pi$ pulses propagation where $m$ is an integer are exclusively relevant to the hyperfine transition $\left|1, F_{1}=1\right\rangle \leftrightarrow\left|4, F_{4}=2\right\rangle$.

3. The area distribution in space for the transition $\left|1, F_{1}=1\right\rangle \leftrightarrow\left|4, F_{4}=2\right\rangle$ characterizes distribution which maximizes the space-entropy with downward peaks corresponding to transition from $2 m \pi$ into $2(m-1) \pi$ in area.
4. Space-entropy $S_{1}(\zeta)$ identifies smeared-out resonances where these transitions are not clearly identified in the behavior of the area $\theta_{1}(\zeta)$ for the first optical transition.

5. The area distributions with negative values are distributions which minimize the space-Bloch entropy.

6. The negative area for field envelope consists of three time sections. The first and second time sections comprise from positive kink and negative anti-kink. The kink and anti-kink are soliton-like waveforms with zero total area. The third section is a generated tail with mainly negative field envelope.

7. The area in $V_{2}$ subsystem with its components $\theta_{3}$ and $\theta_{4}$ for moderate distances show positive and negative peaks, respectively. This reflects the change of sign for the coherence in the Maxwell field equations.

The present paper is devoted to local stabilization of the pulse area and its relation to spatiotemporal Blochmetric distributions. The space-entropy reveals propagation with local stabilization in the pulse area associated with maximum or minimum entropy distributions in space.

\section{Appendix A:}

\section{The time evolution of the density matrix equations}

$$
\begin{aligned}
& \partial_{t} \rho_{1}(t)=-\gamma_{1,1}^{(\text {coll. })} \rho_{1}(t)+\sqrt{\frac{3}{5}} \gamma_{1,1}^{(\text {coll. })} \rho_{2}(t)+\frac{\gamma}{6} \rho_{3}(t)+\frac{1}{2} \sqrt{\frac{5}{3}} \gamma \rho_{4}(t)-2 \frac{v_{1}(t)}{\sqrt{3}} \rho_{5}(t)+2 \sqrt{\frac{5}{3}} v_{2}(t) \rho_{7}(t) \\
& \partial_{t} \rho_{2}(t)=\sqrt{\frac{3}{5}} \gamma_{1,1}^{(\text {coll. })} \rho_{1}(t)-\frac{3}{5} \gamma_{1,1}^{(\text {coll. })} \rho_{2}(t)+\frac{1}{2} \sqrt{\frac{5}{3}} \gamma \rho_{3}(t)+\frac{\gamma \rho_{4}}{2}-2 v_{c_{1}}(t) \rho_{9}(t)+2 v_{c_{2}}(t) \rho_{11}(t) \\
& \partial_{t} \rho_{3}(t)=\left(-\gamma_{4,4}^{(\text {coll. })}-\gamma\right) \rho_{3}(t)+\sqrt{\frac{3}{5}} \gamma_{4,4}^{(\text {coll. })} \rho_{4}(t)+\frac{2 v_{p_{1}}(t)}{\sqrt{3}} \rho_{5}(t)+2 \sqrt{\frac{5}{3}} v_{c_{1}}(t) \rho_{9}(t) \\
& \sqrt{3} \rho_{1}(t)+\sqrt{5} \rho_{2}(t)+\sqrt{3} \rho_{3}(t)+\sqrt{5} \rho_{4}(t)=1, \\
& \partial_{t} \rho_{5}(t)=\frac{1}{\sqrt{3}} v_{p_{1}}(t) \rho_{1}(t)-\frac{1}{\sqrt{3}} v_{p_{1}}(t) \rho_{3}(t)+\left(-\gamma_{3,1 \text { coll. }}^{(1)}-\frac{\gamma}{2}\right) \rho_{5}(t)-\frac{1}{\sqrt{6}} v_{p_{1}}(t) \rho_{13}(t)+\frac{1}{\sqrt{6}} v_{p_{1}}(t) \rho_{15}(t) \\
& +\sqrt{\frac{3}{2}} v_{c_{1}}(t) \rho_{17}(t)-\sqrt{\frac{3}{2}} v_{p_{2}}(t) \rho_{19}(t)
\end{aligned}
$$$$
\partial_{t} \rho_{7}(t)=-\sqrt{\frac{5}{3}} v_{p_{2}}(t) \rho_{1}(t)+v_{p_{2}}(t) \rho_{4}(t)+\left(-\gamma_{3,2 \text { coll. }}^{(1)}-\frac{\gamma}{2}\right) \rho_{7}(t)-\frac{1}{\sqrt{30}} v_{p_{2}}(t) \rho_{13}(t)+\sqrt{\frac{7}{10}} v_{p_{2}}(t) \rho_{16}(t)
$$$$
+\sqrt{\frac{3}{10}} v_{c_{2}}(t) \rho_{17}(t)+\sqrt{\frac{3}{10}} v_{p_{1}}(t) \rho_{19}(t),
$$$$
\partial_{t} \rho_{9}(t)=v_{c_{1}}(t) \rho_{2}(t)-\sqrt{\frac{5}{3}} v_{c_{1}}(t) \rho_{3}(t)+\left(-\gamma_{4,1 \text { coll. }}^{(1)}-\frac{\gamma}{2}\right) \rho_{9}(t)+\sqrt{\frac{7}{10}} v_{c_{1}}(t) \rho_{14}(t)-\frac{1}{\sqrt{30}} v_{c_{1}}(t) \rho_{15}(t)
$$ 


$$
+\sqrt{\frac{3}{10}} v_{p_{1}}(t) \rho_{17}(t)+\sqrt{\frac{3}{10}} v_{c_{2}}(t) \rho_{19}(t)
$$

$$
\begin{aligned}
& \partial_{t} \rho_{11}(t)=-v_{c_{2}}(t) \rho_{2}(t)+v_{c_{2}}(t) \rho_{4}(t)+\left(-\gamma_{4,2 \text { coll. }}^{(1)}-\frac{\gamma}{2}\right) \rho_{11}(t)+\sqrt{\frac{7}{10}} v_{c_{2}}(t) \rho_{14}(t)-\sqrt{\frac{7}{10}} v_{c_{2}}(t) \rho_{16}(t) \\
& \quad+\sqrt{\frac{3}{10}} v_{p_{2}}(t) \rho_{17}(t)-\sqrt{\frac{3}{10}} v_{c_{1}}(t) \rho_{19}(t),
\end{aligned}
$$

$$
\begin{aligned}
& \partial_{t} \rho_{13}(t)=\sqrt{\frac{2}{3}} v_{p_{1}}(t) \rho_{5}(t)+\sqrt{\frac{2}{15}} v_{p_{2}}(t) \rho_{7}(t)-\gamma_{1,1 \text { coll. }}^{(2)} \rho_{13}(t)-\frac{\gamma}{12} \rho_{15}(t)+\frac{1}{4} \sqrt{\frac{7}{3}} \gamma \rho_{16}(t)+\frac{\gamma}{2} \rho_{19}(t) \\
& \quad-\frac{6}{\sqrt{5}} v_{p_{2}}(t) \rho_{25}(t),
\end{aligned}
$$

$$
\begin{aligned}
& \partial_{t} \rho_{14}(t)=-\sqrt{\frac{14}{5}} v_{c_{1}}(t) \rho_{9}(t)-\sqrt{\frac{14}{5}} v_{c_{2}}(t) \rho_{11}(t)-\gamma_{2,2 \text { coll. }}^{(2)} \rho_{14}(t)+\frac{1}{4} \sqrt{\frac{7}{3}} \gamma \rho_{15}(t)+\frac{\gamma}{4} \rho_{16}+\frac{1}{2} \sqrt{\frac{7}{3}} \gamma \rho_{19}(t) \\
& -6 \sqrt{\frac{2}{35}} v_{c_{2}}(t) \rho_{21}(t)+2 \sqrt{\frac{3}{35}} v_{c_{1}}(t) \rho_{23}(t),
\end{aligned}
$$

$$
\partial_{t} \rho_{15}(t)=-\sqrt{\frac{2}{3}} v_{p_{1}}(t) \rho_{5}(t)+\sqrt{\frac{2}{15}} v_{c_{1}}(t) \rho_{9}(t)+\left(-\gamma_{3,3 \text { coll. }}^{(2)}-\gamma\right) \rho_{15}(t)-\frac{6}{\sqrt{5}} v_{c_{1}}(t) \rho_{23}(t),
$$

$$
\partial_{t} \rho_{16}(t)=-\sqrt{\frac{14}{5}} v_{p_{2}}(t) \rho_{7}(t)+\sqrt{\frac{14}{5}} v_{c_{2}}(t) \rho_{11}(t)+\left(-\gamma_{4,4 \text { coll. }}^{(2)}-\gamma\right) \rho_{16}(t)+6 \sqrt{\frac{2}{35}} v_{c_{2}}(t) \rho_{21}(t)+2 \sqrt{\frac{3}{35}} v_{p_{2}}(t) \rho_{25}(t),
$$

$$
\begin{aligned}
& \partial_{t} \rho_{17}(t)=-\sqrt{\frac{3}{2}} v_{c_{1}}(t) \rho_{5}(t)-\sqrt{\frac{3}{10}} v_{c_{2}}(t) \rho_{7}(t)-\sqrt{\frac{3}{10}} v_{p_{1}}(t) \rho_{9}(t)-\sqrt{\frac{3}{10}} v_{p_{2}}(t) \rho_{11}(t)-\gamma_{2,1 \text { coll. }}^{(2)} \rho_{17}(t) \\
& +\sqrt{\frac{6}{5}} v_{p_{2}}(t) \rho_{21}(t)-\frac{1}{\sqrt{5}} v_{p_{1}}(t) \rho_{23}(t)-\frac{1}{\sqrt{5}} v_{c_{2}}(t) \rho_{25}(t),
\end{aligned}
$$

$$
\begin{aligned}
\partial_{t} \rho_{19}(t) & =\sqrt{\frac{3}{2}} v_{p_{2}}(t) \rho_{5}(t)-\sqrt{\frac{3}{10}} v_{p_{1}}(t) \rho_{7}(t)-\sqrt{\frac{3}{10}} v_{c_{2}}(t) \rho_{9}(t)+\sqrt{\frac{3}{10}} v_{c_{1}}(t) \rho_{11}(t)+\left(-\gamma_{4,3 \text { coll. }}^{(2)}-\gamma\right) \rho_{19}(t) \\
- & \sqrt{\frac{6}{5}} v_{c_{1}}(t) \rho_{21}(t)-\frac{1}{\sqrt{5}} v_{c_{2}}(t) \rho_{23}(t)-\frac{1}{\sqrt{5}} v_{p_{1}}(t) \rho_{25}(t),
\end{aligned}
$$

$$
\begin{aligned}
& \partial_{t} \rho_{21}(t)=3 \sqrt{\frac{2}{35}} v_{c_{2}}(t) \rho_{14}(t)-3 \sqrt{\frac{2}{35}} v_{c_{2}}(t) \rho_{16}-\sqrt{\frac{6}{5}} v_{p_{2}}(t) \rho_{17}(t)+\sqrt{\frac{6}{5}} v_{c_{1}}(t) \rho_{19}(t)+\left(-\gamma_{2,4 \text { coll. }}^{(3)}-\frac{\gamma}{2}\right) \rho_{21}(t) \\
&+\sqrt{\frac{2}{7}} v_{c_{2}}(t) \rho_{27}(t)-\sqrt{\frac{2}{7}} v_{c_{2}}(t) \rho_{28}(t),
\end{aligned}
$$

$$
\begin{aligned}
& \partial_{t} \rho_{23}(t)=-\sqrt{\frac{3}{35}} v_{c_{1}}(t) \rho_{14}(t)+\frac{3}{\sqrt{5}} v_{c_{1}}(t) \rho_{15}(t)+\frac{1}{\sqrt{5}} v_{p_{1}}(t) \rho_{17}(t)+\frac{1}{\sqrt{5}} v_{c_{2}}(t) \rho_{19}(t)+\left(-\gamma_{4,1 \text { coll. }}^{(3)}-\frac{\gamma}{2}\right) \rho_{23}(t) \\
& \quad+2 \sqrt{\frac{3}{7}} v_{c_{1}}(t) \rho_{27}(t),
\end{aligned}
$$

$$
\begin{aligned}
& \partial_{t} \rho_{25}(t)=\frac{3}{\sqrt{5}} v_{p_{2}}(t) \rho_{13}(t)-\sqrt{\frac{3}{35}} v_{p_{2}}(t) \rho_{16}+\frac{1}{\sqrt{5}} v_{c_{2}}(t) \rho_{17}(t)+\frac{1}{\sqrt{5}} v_{p_{1}}(t) \rho_{19}+\left(-\gamma_{4,2 \text { coll. }}^{(3)}-\frac{\gamma}{2}\right) \rho_{25}(t) \\
& \quad+2 \sqrt{\frac{3}{7}} v_{p_{2}}(t) \rho_{28}(t)
\end{aligned}
$$

$\partial_{t} \rho_{27}(t)=-2 \sqrt{\frac{2}{7}} v_{c_{2}}(t) \rho_{21}(t)-4 \sqrt{\frac{3}{7}} v_{c_{1}}(t) \rho_{23}(t)+\left(-\gamma-\gamma_{2,2 \text { coll. }}^{(4)}\right) \rho_{27}(t)-\frac{1}{3} \gamma \rho_{28}(t)$,

$\partial_{t} \rho_{28}(t)=2 \sqrt{\frac{2}{7}} v_{c_{2}}(t) \rho_{21}(t)-4 \sqrt{\frac{3}{7}} v_{p_{2}}(t) \rho_{25}(t)+\left(-\gamma-\gamma_{4,4 \text { coll. }}^{(4)}\right) \rho_{28}(t)$. 


\section{References}

[1] S.L. McCall, E.L. Hahn, Phys. Rev. Lett. 18, 908 (1967).

[2] S.L. McCall, E.L. Hahn, Phys. Rev. 183, 457 (1969).

[3] R.E. Slusher, H.M. Gibbs, Phys. Rev. A 5, 1634 (1972).

[4] H.M. Gibbs, R.E. Slusher, Phys. Rev. A 6, 2326 (1972).

[5] G.L. Lamb, Rev. Mod. Phys. 43, 99 (1971).

[6] V.E. Zakharov, A.E. Shabat, Sov. Phys. JETP 34, 62 (1972).

[7] D.V.J. Novitsky, J. Phys. B At. Mol. Phys. 47, 095401 (2014).

[8] T.W. Barnard, Phys. Rev. A 7, 373 (1973).

[9] M.J. Ablowitz, H. Segur, Solitons and the Inverse Scattering Transform, Siam, Philadelphia 1981.

[10] P.J. Caudrey, J.C. Eilbeck, Phys. Lett. 62, 65 (1977).

[11] A.M. Alhasan, J. Fiutak, W. Miklaszewski, Z. Phys. B 88, 349 (1992).

[12] A.M. Alhasan, J. Czub, W. Miklaszewski, Phys. Rev. A 80, 033809 (2009).

[13] P. Siddons, J. Phys. B At. Mol. Phys. 47, 093001 (2014).

[14] C.R. Stroud Jr., D.A. Cardimona, Opt. Commun. 37, 221 (1981).

[15] M. Matusovsky, B. Vaynberg, M. Rosenbluh, Phys. Rev. Lett. 77, 5198 (1996).

[16] M. Materassi, Entropy 18, 304 (2016).
[17] A.B. Naim, Entropy 19, 48 (2017).

[18] P. Garbaczewski, Entropy 7, 253 (2005).

[19] A.M. Alhasan, Entropy 17, 5920 (2015).

[20] A.M. Kamchatnov, Phys. Lett. A 202, 54 (1995).

[21] D.A. Steck, Rubidium 87 D Line Data, Los Alamos National Laboratory, data sheet.

[22] W. Maichen, F. Renzoni, I. Mazets, E. Korsunsky, L. Windholz, Phys. Rev. A 53, 3444 (1996).

[23] A.F. Huss, E.A. Korsunsky, L. Windholz, J. Mod. Opt. 49, 141 (2002).

[24] L. Windholz, Act. Phys. Pol. A 97, 259 (2000).

[25] J. Fiutak, J. Van Kranendonk, J. Phys. $B$ At. Mol. Phys. 13, 2869 (1980).

[26] C.Y. Yinhe, W. Tung, J.B. Gao, V.A. Protopopescu, L.M. Hively, Phys. Rev. E 70, 046217 (2004).

[27] A. Vardi, M. Shapiro, Phys. Rev. A 62, 025401 (2000).

[28] M.E. Patino, R.S. Berry, Phys. Rev. A 34, 4728 (1986).

[29] H.-P. Breuer, Phys. Rev. A 68, 032105 (2003).

[30] S. Sen, M.R. Nath, T.K. Dey, G. Gangopadhyay, Ann. Phys. 327, 224 (2012).

[31] S.K. Goyal, B.N. Simon, R. Singh, S. Simon, J. Phys. A Math. Theor. 49, No. 16 (2016).

[32] P. Jizba, H. Kleinert, M. Shefaat, Physica A 391, 2971 (2012).

[33] J.F. Bercher, Phys. Lett. A 373, 3235 (2009).

[34] E.J. Robinson, Phys. Rev. A 24, 2239 (1981).

[35] A. Bambini, P.R. Berman, Phys. Rev. A 23, 2496 (1981). 